\title{
Visión enfermera de las necesidades psicosociales de los cuidadores de personas en tratamiento con Diálisis Peritoneal
}

Premio Baxter a la Investigación sobre la Diálisis Peritoneal

Lola Andreu Periz* - Paqui Gruart Armangué ** - Lia Tamar Sánchez-Salido***

* Departamento de E nfermería F undamental y M édico Quirúrgica. U niversidad de Barcelona.

** Servicio de Nefrología Hospital Universitario de Bellvitge. Barcelona

*** Unidad de Cuidados Intensivos. Hospital Universitario de B ellvitge. Barcelona

\section{Resumen}

Debido a la edad o a patologías interrecurrentes muchas personas en programa de Diálisis Peritoneal Ambulatoria, necesitan total o parcialmente de la ayuda de un cuidador por lo que la visión de los profesionales respecto al cuidador y sus necesidades es fundamental para ofrecer unos cuidados de salud adecuados.

Con el objetivo de identificar y describir la visión de la enfermería responsable de los programas de diálisis peritoneal respecto a las necesidades psicosociales de los cuidadores principales de las personas sometidas a esta forma de tratamiento se realizó a 7 profesionales de enfermería nefrológica entrevistas semiestructuradas con un guión previo con preguntas abiertas. EI análisis cualitativo de sus respuestas sugiere que para poder implementar eficazmente la participación familiar en los cuidados es imprescindible la organización personalizada y el soporte institucional; que la atención a la familia es una de las actividades de enfermería que no se suele valorar y para la que

\section{Correspondencia: \\ Lola Andreu \\ Escuela de Enfermería. Campus de Bellvitge \\ $\mathrm{C} / \mathrm{F}$ eixa Llarga $\mathrm{s} / \mathrm{n}$ \\ 08907 Hospitalet de Llobregat (Barcelona)}

los responsables de la DP se sienten en ocasiones solos y faltos de recursos. Para los grandes problemas, como el cansancio del cuidador, se apuntan soluciones como buscar lugares fuera del hogar donde se le pueda realizar al paciente la técnica temporalmente.

Palabras clave:

DIÁLISIS PERITONEAL CONTINUA AMBULATORIA CUIDADOR

ANÁLISIS CUALITATIVO

NE CESIDADE S PSICOSOCIALES

Nursing view of the psychosocial needs of carers of people undergoing Peritoneal Dialysis

\section{Abstract}

Due to age and recurrent pathologies, many people on the Ambulatory Peritoneal Dialysis programme need the full or part-time assistance of a carer, and therefore the view of professionals in relation to the carer and his or her needs is fundamental in order to offer adequate healthcare.

In order to identify and describe the view of nursing staff responsible for the peritoneal dialysis programmes with regard to the psychosocial needs of the main carers of people who undergo this 
form of treatment, semi-structured interviews of 7 renal nursing professionals were carried out, using a guideline with open questions. The qualitative analysis of their responses suggests that in order to implement family participation in care efficiently, personalized organization and institutional support are essential; attention to the family is one of the nursing activities that is not usually valued and for which staff responsible for PD sometimes feel alone and with insufficient resources. For major problems, such as tiredness of the carer, solutions were indicated such as seeking places outside the home where the patient can undergo the technique temporarily.

\section{KEY WORDS \\ CONTINUOUS AMBULATORY PERITONEAL \\ DIALYSIS \\ CARER \\ QUALITATIVE ANALYSIS \\ PYSCHOSOCIAL NEEDS}

\section{Introducción}

La Diálisis Peritoneal (DP) puede ofrecer a la persona gran autonomía, aunque, en ocasiones, se indica en situaciones en las que el paciente, precisamente por su falta de autonomía y labilidad, no es candidato a otro tipo de terapia como la hemodiálisis o el trasplante. E sto hace que de la población en DP, un porcentaje significativo sean personas dependientes que precisan de un cuidador para cubrir total o parcialmente sus necesidades, además de realizar la técnica y los cuidados específicos que ésta conlleva. La enfermería nefrológica se encuentra inmersa en la paradoja que plantea el ofrecer una técnica muy adecuada para personas independientes, ya que puede ser autoadministrada, pero que muchas veces debe realizarse a pacientes dependientes cuyo posible cuidador puede ser alguien mayor, con el déficit propio de la edad avanzada, o bien ser una persona joven pero con una disponibilidad limitada, por tener otras cargas familiares o sociales que limiten su rol de cuidador.

La visión de los profesionales respecto al cuidador y sus necesidades es fundamental para ofrecer unos cuidados de salud adecuados. Aunque son numerosos los estudios que describe la percepción de los pacientes y familia respecto a la atención sanitaria recibida ${ }^{1,2}$, pocos se centran en descubrir que piensa la enfermería sobre el cuidador y cuál es su rol respecto a éste ${ }^{3}$.

EI mejor conocimiento de la relación enfermera-cuidador sin duda contribuirá a una mejor atención a la persona en DP, para esto puede ser útil apoyarse en una investigación cualitativa, ya que es un tipo de investigación que trata de identificar la naturaleza profunda de las realidades, su sistema de relaciones; es aquella que estudia la relación en contextos estructurales y situacionales ${ }^{4}$.

Aunque la enfermería nefrológica suele utilizar la investigación cuantitativa como método de investigación, puede beneficiarse extraordinariamente de la investigación cualitativa ya que al examinar la naturaleza del cuidado se hacen evidentes cuestiones que ni los experimentos ni las mediciones propias de la investigación cuantitativa pueden responder ${ }^{5}$. Cuidar es una actividad humana que tiene aspectos invisibles e intangibles; es un proceso en el cual la interacción con la persona a la que se cuida es fundamental, por lo que plantea preguntas del tipo: ¿cómo investigar la interacción entre el que cuida y el que es cuidado?, ¿cómo formalizar la buena práctica del cuidado de enfermería? ${ }^{4}$. Abordar estas y otras cuestiones relacionadas con el cuidado de paciente renal abren un amplio campo de investigación del que podemos obtener múltiples beneficios ya que el conocer mejor temas tan complejos como las reacciones humanas facilita el establecer bases de acción para seguir en la práctica profesional| ${ }^{4,5}$.

Objetivo: Identificar y describir cual es la visión de la enfermería responsable de los programas de diálisis peritoneal respecto a las necesidades psicosociales de los cuidadores principales de las personas sometidas a esta forma de terapia

\section{Material y método}

E ste estudio se llevó a cabo en el área sanitaria de B arcelona para lo que se realizó un muestreo de conveniencia de 7 profesionales de enfermería nefroló- 
gica responsables de los programas de DP de los principales centros de salud del área, cuya experiencia en este campo era de entre 2 y 20 años. P ara la recogida de datos se realizaron 7 entrevistas que tuvieron una duración media de 55 minutos, llevándose a cabo en un periodo de cuatro semanas, que se inicio en marzo de 2005. El número de sujetos fue suficiente para llegar a la comprensión del fenómeno interrogado y explorar el rol de enfermería en relación al grupo de cuidadores; pues no se buscaron generalizaciones, pero sí generalidades de los discursos analizados. La elección de los entrevistados se basó en su lugar de trabajo, en la experiencia y trayectoria profesional junto a una fácil accesibilidad.

Se realizaron entrevistas semiestructuradas, es decir se preparó un guión previo de preguntas abiertas que en ningún caso siguieron una secuencia sistemática y rígida, procurando el investigador ejercer un control mínimo sobre ellas. E I objetivo de estas entrevistas era conocer comportamientos, experiencias, opiniones, conocimientos y otros aspectos relacionados con el tema a tratar4. Las preguntas estaban centradas en la práctica diaria de los profesionales y su punto de vista sobre el tema a tratar (tabla 1). Después de cada entrevista se tomaron notas sobre su desarrollo con el fin de facilitar la reflexión sobre la misma ya que la recogida de datos y su análisis se realizaron simultáneamente apoyándose en la bibliografía consultada ${ }^{4-6}$. Los profesionales entrevistados fueron informados detalladamente sobre la investigación para que decidieran su participación en el estudio, garantizando la confidencialidad respecto a su identificación en publicaciones.

\section{Resultados}

Del análisis del discurso de los participantes se definieron cuatro categorías mayores o temáticas, que se interpretaron con apoyo de la literatura revisada. Frases significativas de los entrevistados que apoyan el análisis se reseñan en cursiva.

1. Características deseables en la persona que se somete a DP. Todos los participantes definieron como candidato "ideal" para someterse a tratamiento con DP a una persona autónoma y capaz de realizar por si mismo el tratamiento. No obstante, todos tenían experiencia con personas a las que un cuidador rea- lizaba todo el proceso. Reconocían que aunque ésta no era una situación deseable los resultados clínicos eran extrapolables a los de los pacientes autónomos.

“EI paciente en DP debería ser autónomo y capaz de llevar por si sólo la técnica. Lo demás son malas soluciones". "Aunque sería deseable que el paciente pudiera desenvolverse por si mismo, muchas veces es inviable, sólo la ayuda de un familiar hace posible la técnica". "L as dos opciones son posibles; si la persona no es autónoma, un familiar puede hacerse cargo de todo el proceso". "La verdad es que si comparas a pacientes dependientes o independientes de la misma edad, no tiene ni más peritonitis ni otros problemas unos que otros".

Respecto a otras características como edad, situación familiar etc. no hubo consenso entre los entrevistados, para unos podía ser la mejor candidata una persona de mediana edad, con una situación familiar estable (con pareja), mientras que para otros, los jóvenes serían los ideales ya que podían alcanzar mayor independencia a la espera de un trasplante. No obstante todos consideraron la DP como una opción válida para cualquier persona con IRCT y consideraron un error que la DP sea en ocasiones la "última posibilidad" en vez de "una de las mejores opciones".

"La DP puede ofrecer una gran calidad de vida, pero muchas veces no se le ofrece a los pacientes". "Hay pacientes a los que nunca se les ha hablado de la DP y serían excelentes candidatos"

2. Aspectos organizativos de la DP en relación con la autonomía del paciente. No se consideró una gran dificultad para la organización que el paciente en DP no fuera autónomo en la realización del tratamiento. E I tiempo de entrenamiento podía ser similar, tanto si se adiestraba a una persona autónoma como a un cuidador y el seguimiento no planteaba diferencias. E s deseable una interrelación entre el paciente y un familiar con respecto al entrenamiento, y se considera oportuno que aunque el paciente sea autónomo, un familiar conozca los aspectos básicos de la técnica.

"No hay diferencia en entrenar a una persona autónoma $o$ a un cuidador, lo que importa es la motivación". "Siempre hago venir a un familiar para explicarle los aspectos más relevantes de la técnica". 
"Les digo que venga un familiar para que aprenda por si un día ellos no pueden hacerse la DP".

Los entrevistados consideran muy valiosa la relación directa con el paciente aunque éste dependa de un cuidador y procuran dirigirse siempre a él, incluso ante instrucciones que deban dar al cuidador.

"A unque sea una persona muy mayor o con dificultades cognitivas siempre procuro dirigirme a él y explicarle todos lo referente a su salud". "Me sentiría mal si hablara de mi paciente a sus espaladas". "Aunque necesite ayuda, el paciente es el que debe conocer todo lo que ocurre"

Casi todos los pacientes autónomos recurren a la ayuda de un familiar para tareas relacionadas con la técnica como el almacenaje del material o la cura del orificio. E sto parece positivo, siempre que no se abuse, ya que se considera que el paciente es responsable de la técnica y no tiene que descargar este proceso en su familia.

"Insisto para que sea el paciente el que haga todo lo que pueda, pero a veces se apoyan demasiado en la familia". "L a peritoneal se vive en la familia, es lógico que ésta ayude".

La atención de la enfermería se centra en paciente y aunque se preste atención al cuidador es desde la perspectiva de darle el apoyo necesario para que cuide mejor a su familiar. Destaca el papel que el cuidador tiene como informador, en este y otros sentidos el cuidador adquiere un papel de colaborador de la enfermería.

"A veces te cuentan cosas que el paciente esconde". "En la visita dicen: no le haga caso que sí come con sal"." El cuidador es el se fija en estos detalles".

3. Actitud del cuidador desde la perspectiva de la enfermera. Los cuidadores pueden adoptar actitudes muy diversas dependiendo de su perfil y grado de implicación. La gran diferencia estriba en que el cuidador haya elegido libremente la opción de atender a su familiar o se haya visto obligado a ello como última alternativa. Otros factores determinantes son el grado de parentesco y la edad del cuidador.
"Si el cuidador es la esposa, va mucho mejor que si es la hija o la nuera". "A veces la mujer está casi tan mayor como el paciente". "Si el cuidador es una persona joven, la DP le priva mucho de libertad". "A veces al cuidador no se le plantea otra opción y esto siempre es duro". "Se debe procurar no presionar al cuidador".

Cabe destacar que en general la figura del cuidador se aborda desde una perspectiva de género y se suele utilizar "la esposa", "la mujer", "ella" al referirse al cuidador aunque que en los casos menos frecuentes en que el cuidador es un hombre lo hace " $\tan$ bien como una mujer".

Si el cuidado de la persona en DP es responsabilidad de personas diferentes a la pareja, se suele optar por la solución de compartir el cuidado entre varios miembros de la familia. E sto parece a los entrevistados un mal menor porque las posibilidades de error aumentan, pero también consideran que puede ser la única forma de la familia pueda disponer de cierta libertad. E $n$ este caso se procura entrenar a todos los cuidadores, aunque no siempre se consigue, por falta de disponibilidad de estos o porque la enfermera desconoce que la familia ha decidido compartir el trabajo sin comunicárselo previamente.

"Siempre procuramos entrenar a todas las personas que van a hacer la técnica, pero a veces sólo pueden venir una vez a repasar". "La hija de la paciente enseñó a los nietos para que entre todos la hicieran, sólo vinieron una vez cada uno, pero lo hacían bien". "Tú enseñas a un cuidador principal, pero luego, en su casa, ellos hacen lo que les parece."

La actitud del cuidador puede ir desde la resignación a una motivación extrema, que le lleve a ser muy meticuloso y a sentirse responsable de todo lo que ocurra, sobreprotegiendo al paciente, Muchas veces busca una "alianza" con el personal sanitario para convencer a su familiar de que realice un adecuado cumplimiento terapéutico. Si tiene problemas los manifiestan mayoritariamente a la enfermera.

4. Mecanismos de apoyo al cuidador. E n general la sociedad y las instituciones prestan atención sólo al paciente, olvidando al cuidador y sus problemas; se le utiliza sin reconocer el importante papel que tiene y 
el esfuerzo que realiza. E I cansancio, la ansiedad, el temor que en muchas ocasiones manifiesta el cuidador sólo suele detectarlo la enfermera responsable de $D P$, que en ocasiones, es su única válvula de escape. E s necesario valorar con frecuencia la actitud del cuidador, ofrecerle apoyo si aparecen problemas y proporcionarle períodos de descanso, pero para esto se ha de contar con apoyo institucional. El ofrecer una cantidad en metálico en concepto de gratificación es una opción bien valorada por los entrevistados.

"M uchas veces el cuidador no puede más". "Delante del enfermo ponen buena cara pero a veces te confían que están agotados". "Es muy duro tener esa responsabilidad toda la vida". "Se les ha de animar como se puede". "Tendría que haber un sistema para que los cuidadores descansarán". "Sólo una temporada de descanso puede aliviarlos".

\section{Discusión}

Como comentarios a nuestro análisis cabe destacar que salvo en aspectos muy puntuales hubo una gran concordancia entre las opiniones de los entrevistados, lo que indica que la enfermería de DP tiene metas comunes y una visión de los problemas muy similar.

La persona enferma y su familia forman un todo en el que producen intercambios de ayuda que permiten manejar las situaciones difíciles provocadas por la enfermedad y su tratamiento. E sta ayuda se conceptualiza como un recurso evidente que permite a la enfermería realizar mejor su rol. Numerosos autores avalan la importancia de la familia como proveedora de cuidados ${ }^{1,2,7}$.

E I entramado familiar puede ser muy complejo, siendo un factor esencial la motivación para cuidar al enfermo. Por esto, los criterios a la hora de elegir un cuidador no pueden ser rígidos, debiendo adaptarse los programas de DP a las necesidades del grupo familiar. Se tiende a considerar al cuidador más como un colaborador que como un receptor de cuidados, por lo que es difícil detectar sus necesidades y ayudarle a cubrirlas ${ }^{7}$. Queda patente que, como sugieren otros autores, para poder implementar eficazmente la participación familiar en los cuidados es imprescindible la organización personalizada y el soporte institucional ${ }^{1,2}$. Por otra parte, como refiere la bibliografía, la atención a la familia es una de las actividades de enfermería que no se suele valorar y para las que los responsables de la DP se sienten en ocasiones solos y faltos de recurso ${ }^{8-11}$. Para los grandes problemas, como el cansancio del cuidador, se apuntan soluciones como buscar lugares fuera del hogar donde se le pueda realizar al paciente la técnica temporalmente, aunque esto es poco factible, teniendo en cuenta la actual organización de la mayor parte de unidades nefrológicas.

Se debe profundizar en el conocimiento de las necesidades del cuidador como parte integrante del plan de cuidados y demandar de las instituciones el apoyo necesario para hacerlo. Entrevistas en profundidad con los cuidadores pueden poner de manifiesto necesidades encubiertas y ampliar el campo de actuación de la enfermera. E ste y otros métodos cualitativos de investigación pueden ser herramientas muy útiles para la investigación en enfermería, ya que permiten el descubrimiento de fenómenos nuevos y la descripción lo que facilita, que se renueven y amplíen los esquemas mentales.

\section{Agradecimientos}

A Ma J osé Arnau, Laura Tarrats, Mercè Perez, Ma E lisa Vilaplana, Ferran Vilarnau y Antonia Vives, cuyas reflexiones han hecho posible este trabajo.

\section{Bibliografía}

1. Celma M. Cuidadores informales en el medio hospitalario. Revista Rol de Enfermería 2001; 24(78):503-501.

2. E scudero B, Díaz E, Pascual 0. Cuidadores informales. Necesidades y ayuda. Revista Rol de Enfermería 2001; 24 (3):183-189.

3. Contel J C, González M. Reflexión sobre la implementación de programas de atención domiciliaria. E nfermería Clínica 1995; 5(5): 205-211. 
4. Cabrero García L, Richart Martínez M. E I debate investigación cualitativa frente a investigación cuantitativa. Enfermería Clínica 1996; 6: 212217.

5. M ontiel, M . La investigación cualitativa en enfermería nefrológica. Revista de la Sociedad E spañola de Enfermería Nefrológica 2002; (6)2:52-56.

6. Abdellah FG, Levine E. Preparing Nursing Research for the 21 st Century. Evolution. Methodologies, Chalges. Springer: New York; 1994.

7. Álvarez Cáceres R. El método científico en las ciencias de la salud. L Las bases de la investigación biomédica. Madrid: Díaz de Santos; 1996.
8. Aznar M I, F leming V, Watson H, Narvaiza MJ . Necesidades psicosociales de los cuidadores informales de las personas con esquizofrenia: exploración del rol profesional de enfermería. E nfermería Clínica 2004; 14(5): 286-93.

9. De la Cuesta C. Creación de contextos: trabajo intangible en enfermería comunitaria. Revista Rol de E nfermería 1994; 195:13-19.

10. García Barbero M. From hospitals to home health care: an alternative requiring careful thinking. Copenhague: WHO; 1997.

11. Coleman B. E uropean models of long-term care in home community. Internal Journal Health Service 1995; 25: 458-74.

\section{Preguntas guía}

¿Quien gestiona, coopera, diseña y ejecuta las actividades en la DP?

¿Cuál es le perfil "ideal” de la persona en tratamiento con DP?

¿Cómo influye el tratamiento con DP en el contexto familiar?

¿Qué funciones posee o se delegan al cuidador principal?

¿Qué se entiende como necesidades del cuidador?

¿Cómo se identifican estas necesidades?

Tabla 1. Preguntas guía para la entrevista. 